\title{
Der Honorararzt: Arbeitnehmer oder selbstständige Tätigkeit? - Aktuelle Rechtsentwicklungen
}

\section{Einführung}

Das Honorararztwesen hat angesichts des zunehmenden Ärztemangels in Krankenhäusern an Bedeutung gewonnen. Speziell in ländlichen Gebieten, in denen Versorgungsengpässe die Krankenhäuser vor besondere Herausforderungen stellen, sind Honorarärzte prinzipiell aus dem Klinikalltag nicht mehr wegzudenken. Dieser Lebenswirklichkeit hat jüngst auch der Gesetzgeber mit der Einführung des $\S 2$ Abs. 1 S. 1 KHEntgG Rechnung getragen, da die Frage ob und in welchem Umfang Ärzte, die weder Belegärzte, noch hauptamtlich im Krankenhaus tätig sind, generell zur Erbringung von Krankenhausleistungen für bestimmte Untersuchungen und Behandlungen hinzugezogen werden können, sehr umstritten war. Der Gesetzgeber hat diese Frage nunmehr dahingehend geklärt, dass Krankenhausleistungen - in den Grenzen des §20 Ärzte-ZV - „auch durch nicht fest angestellte [...] Ärzte“ erbracht werden können ( $§ 2$ Abs. 1 S. 1 KHEntgG).

Folglich ist der Einsatz von Honorarärzten nach dieser Norm vom Grundsatz her zuläs$\mathrm{sig}^{1}$. Die Leistung muss sich aber weiterhin unter Würdigung des Gesamtzusammenhangs als Leistung des Krankenhauses darstellen ${ }^{2}$. Das BSG ${ }^{3}$ hat diesbezüglich festgestellt, dass es der gesetzliche Regelfall sei, dass Krankenhäuser mit eigenem Personal arbeiteten. Dies bedeutet im Umkehrschluss, dass die Grenze seines Leis-

1 OVG Lüneburg, GesR 2013, 495; Münzel, PKR 2016, 4 (6f.); vgl. auch BT-Drs. 17/9992, S. 26 f.; a.A. LSG BW, NZS 2013, 501 (503 ff.); sich anschließend SG München, Urt. v. 10.3.2016, Az.: S 15 R 1782/15; in einer aktuellen Entscheidung des BSG (GesR 2016, 240), der ein Sachverhalt vor Inkrafttreten des § 2 Abs. 1 S. 1 KHEntgG n. F. zu Grunde liegt, äußert das Gericht ebenfalls allgemeine Bedenken bzgl. der Frage der Zulässigkeit von Krankenhausbehandlungen durch nicht angestellte Ärzte.

2 Möller/Makoski, GesR 2012, 647 (649).

3 BSG, ZMGR 2011, 300 (307). tungserbringerstatus‘ dann überschritten ist, wenn Leistungen nicht mehr regelmäßig mit angestelltem Personal, sondern mit externen Ärzten erbracht werden ${ }^{4}$. Hieraus folgert er, dass der Leistungszukauf einen Ausnahmecharakter besitze. Daher erbringt das Krankenhaus ab einem bestimmten Volumen der für sich genommenen zulässigen Einzelzukäufe die Gesamtleistungen nicht mehr im Rahmen seines Leistungserbringerstatus‘.

Gleichwohl bleibt eine Reihe von Rechtsfragen unbeantwortet ${ }^{5}$. Neben den offenen Fragen nach einer Beschränkung der Tätigkeit des Honorararztes auf „ergänzende oder unterstützende“ Leistungen ${ }^{6}$ sowie der Angemessenheit der Vergütung ${ }^{7}$ trifft $\S 2$ Abs. 1 S. 1 KHEntgG keine Aussage zur sozi-

4 In diesem Sinne wohl auch Stollmann, NZS 2011, 684 (685); a.A. Seiler, NZS 2011, 410 (413).

5 Die kontrovers diskutierte Frage (zum Streitstand siehe Jenschke, GesR 2015, 136 (138f.)), ob Honorarärzte Wahlleistungen i. S. d. $\S 17$ Abs. 1 S. 1 KHEntgG erbringen und abrechnen dürfen, hat der BGH (MedR 2015, 120) abschlägig beschieden, da die Wahlarztkette in $\S 17$ Abs. 3 KHEntgG abschließend und eine Öffnung für Honorarärzte nicht vorgesehen sei. Die Vorschrift sei eine dem Schutz des Privatpatienten dienende zwingende preisrechtliche Norm, von der auch nicht durch individuelle Vergütungsvereinbarung abgewichen werden könne. Derartige Vergütungsvereinbarungen seien deswegen gem. §134 BGB nichtig.

6 Bejahend zur alten Rechtlage BSG, ZMGR 2011, 300 (306 ff.); BSG, GesR 2016, 240 (243 f.); LSG Sachsen, GesR 2008, 548 (549f.); a.A. Seiler, NZS 2011, 410 (413) sowie Clemens, MedR 2011, 770 (781); i.E. auch Möller/ Makoski, GesR 2012, 647 (649).

7 Die Frage nach der Angemessenheit der Vergütung stellt sich speziell vor dem Hintergrund des Verbotes der Zuweisung gegen Entgelt ( $\S 73$ Abs. 7 SGB V, $\S 31$ Abs. 1 MBOÄ, §31a KHGG NRW, sog. kick-back-Zahlung), wenn die betreffenden Honorarärzte zu Einweisern des Krankenhauses zählen. Mit Blick auf $\S \S 299 a-300$ StGB (Bestechung und Bestechlichkeit im Gesundheitswesen) bestehen nunmehr auch strafbarkeitsrechtliche Risiken.

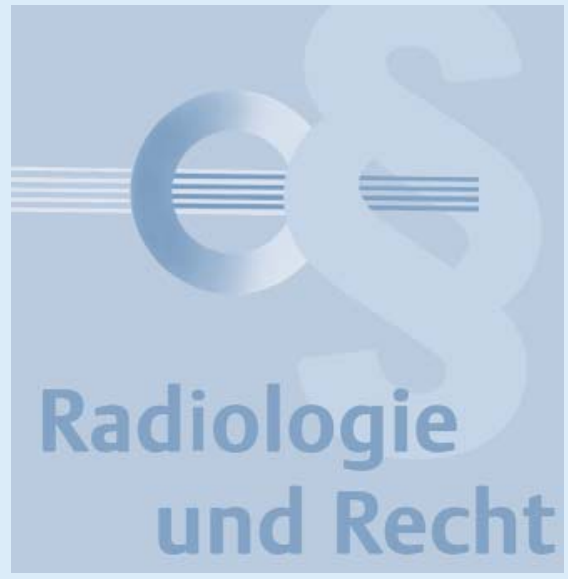

alversicherungs- und arbeitsrechtlichen Statusbewertung des Honorararztes. Vor dem Hintergrund vermehrter Betriebsprüfungen gem. §28 p SGB IV durch die Sozialversicherungsträger wird die Frage, ob bzw. in welchen Fällen der im Krankenhaus tätige Honorararzt tatsächlich freiberuflich tätig ist oder ob de facto nicht doch ein sozialversicherungspflichtiges Beschäftigungsverhältnis vorliegt, kontrovers diskutiert. Neben der Gefahr erheblicher Nachzahlungen durch den Krankenhausträger gewinnt diese Problematik mit Blick auf §266a StGB (Vorenthalten und Veruntreuen von Arbeitsentgelt) unmittelbar auch strafrechtliche Relevanz (vgl. auch Strafbarkeit gem. §370 AO wegen Steuerhinterziehung). Weiterhin können sich aus der arbeitsrechtlichen Feststellung der Arbeitnehmereigenschaft eines Honorararztes Rechtsfolgen wie die Anwendung des Kündigungsschutzgesetzes, des Bundesurlaubsgesetzes oder von Tarifverträgen ergeben. Zahlreiche sozialgerichtliche Entscheidungen aus der jüngeren Vergangenheit belegen, dass die Statusbewertung des Honorararztes erhebliche Schwierigkeiten bereitet. Anhand eines aktuellen Urteils des Landessozialgerichtes (LSG) BadenWürttemberg ${ }^{8}$, welches eine honorarärztlich tätige Radiologin betraf, sollen die aktuellen Anforderungen der Rechtsprechung an die honorarärztliche Tätigkeit nachfolgend dargestellt und kritisch gewürdigt werden. 


\section{Begriff des Honorararztes}

Nach ihrer Definition sind Honorarärzte Ärzte, die im Krankenhaus Leistungen erbringen, ohne Arbeitnehmer oder Beschäftigter zu sein, und dafür vom Krankenhaus ein zuvor vereinbartes Honorar erhalten ${ }^{9}$. Ähnlich fällt die Einordnung durch den $\mathrm{BGH}^{10}$ aus. Unter einem Honorararzt ist ein Facharzt zu verstehen, der im stationären und/oder ambulanten Bereich des Krankenhauses ärztliche Leistungen für den Krankenhausträger erbringt, ohne bei diesem angestellt oder als Belegarzt oder Konsiliararzt tätig zu sein. Er wird zeitlich befristet freiberuflich auf Honorarbasis tätig, wobei das Honorar mit dem Krankenhausträger frei und unabhängig von den Vorgaben der GOÄ vereinbart wird.

Im Rahmen des mit dem Krankenhaus geschlossenen Vertrages wird der Arzt als dessen Erfüllungsgehilfe i. S. d. § 278 BGB tätig, d. h. er erfüllt dessen Vertragspflichten, die aus dem Behandlungsvertrag zwischen Patient und Krankenhausträger resultieren. Schließt er hingegen einen eigenen Behandlungsvertrag gem. §630a BGB mit dem Patienten $a b$ und rechnet seine Leistungen selbst gegenüber der Kassenärztlichen Vereinigung ab, wird er nicht als Honorararzt, sondern regelmäßig als Belegarzt tätig.

\section{Statusbewertung des Honorararztes im Krankenhaus}

Eine höchstrichterliche Entscheidung zu der Frage, nach welchen konkreten Maßstäben der sozialversicherungs- und arbeitsrechtliche Status des Honorararztes zu beurteilen ist, steht bislang noch aus. Die von der (obergerichtlichen) Judikatur entwickelte bisherige Kasuistik ist jedoch mitunter fragwürdig und steht bisweilen - auch unter Berücksichtigung der jeweiligen Lebenssachverhalte - im Widerspruch zueinander. Im Gegensatz zur arbeitsgerichtlichen Judikatur lässt die sozialgerichtliche Rechtspre-

9 DKG, Honorarärzte im Krankenhaus - eine arbeits- und sozialversicherungsrechtliche Standortbestimmung, 2014.

10 BGH, MedR 2015, 120. chung oftmals die Berücksichtigung der Besonderheiten einer (honorar-) ärztlichen Tätigkeit vermissen. Mitunter wird sogar als Leitsatz behauptet, dass im Krankenhaus tätige Ärzte regelmäßig abhängig beschäftigt seien ${ }^{11}$.

\section{Arbeitnehmerbegriff nach der höchstrichterlichen Recht- sprechung}

Ausgangspunkt für die rechtliche Beurteilung ist $\S 7$ Abs. 1 SGB IV. Demgemäß ist „Beschäftigung [...] die nichtselbständige Arbeit, insbesondere in einem Arbeitsverhältnis. Anhaltspunkte für eine Beschäftigung sind eine Tätigkeit nach Weisungen und eine Eingliederung in die Arbeitsorganisation des Weisungsgebers. “ Den Begriff der Beschäftigung hat das BSG in zahlreichen Entscheidungen näher konkretisiert: „Nach der ständigen Rechtsprechung des [BSG] setzt eine Beschäftigung voraus, dass der Arbeitnehmer vom Arbeitgeber persönlich abhängig ist. Bei einer Beschäftigung in einem fremden Betrieb ist dies der Fall, wenn der Beschäftigte in den Betrieb eingegliedert ist und dabei einem Zeit, Dauer, Ort und Art der Ausführung umfassenden Weisungsrecht des Arbeitgebers unterliegt. Demgegenüber ist eine selbstständige Tätigkeit vornehmlich durch das eigene Unternehmerrisiko, das Vorhandensein einer eigenen Betriebsstätte, die Verfügungsmöglichkeit über die eigene Arbeitskraft und die im wesentlichen frei gestaltete Tätigkeit und Arbeitszeit gekennzeichnet. Ob jemand abhängig beschäftigt oder selbstständig tätig ist, hängt davon $a b$, welche Merkmale überwiegen [...]. Maßgebend ist stets das Gesamtbild der Arbeitsleistung. " 12 Zu beachten ist allerdings, dass sich der jeweilige Vertragstyp aus dem praktizierten Geschäftsinhalt ergibt. Widersprechen sich die vertraglichen Abreden und die gelebte Vertragsdurchführung, ist für die Bestimmung der Rechtsnatur des Rechtsverhältnisses Letztere maßgeblich $^{13}$.

11 LSG BW, Urt. v. 27.4.2016, Az.: L 5 R 852/14R.

12 BSG, GmbHR 2006, 645 (646); vgl. auch BSG, SGb 2013, 364.

13 St. Rspr., etwa BSG, NZS 2001, 414.
Demgegenüber hat das LSG Baden-Württemberg ${ }^{14}$ einen gänzlich anderen Ansatz gewählt. Es vertritt die Ansicht, dass die ärztliche Tätigkeit in einem abhängigen Beschäftigungsverhältnis erfolgt sein muss, weil die Tätigkeit aus Rechtsgründen nur so erfolgen könne. Das Krankenhaus könne nur Vergütungen der gesetzlichen Krankenversicherung in Anspruch nehmen und ihren Versorgungsauftrag in rechtlich zulässiger Weise erfüllen, wenn die ärztliche Leistung im Rahmen eines Beschäftigungsverhältnisses oder einer Kooperation mit einem niedergelassenen Arzt erbracht wird. Damit wäre die Beschäftigung von Honorarärzten per se ausgeschlossen, zumindest soweit diese nicht auch gleichzeitig über eine Niederlassung verfügen. Vertragstypologisch könne nicht sein, was krankenhausrechtlich nicht gestattet sei. Gegen diese Sichtweise des 5. Senates spricht indes, dass das KHEntgG lediglich die Vergütungsansprüche von Krankenhäusern regelt, aber keine Aussagen zum sozialversicherungsrechtlichen Status von im Krankenhaus tätigen Personen trifft ${ }^{15}$. Sie ist daher abzulehnen.

\section{Kriterien der Weisungsgebunden- heit und der Eingliederung in die Betriebsorganisation}

Der Beschäftigungsbegriff wird mithin nicht durch eine Summe von abschließenden Kriterien definiert, sondern als Typus dargestellt. Demgemäß besteht für die Gerichte ein großer Beurteilungsspielraum. Charakteristisches Hauptmerkmal eines Beschäftigungsverhältnisses ist nach der Rechtsprechung die (fachliche, örtliche und zeitliche) Weisungsgebundenheit gegenüber dem Arbeitgeber. Derartige Anhaltspunkte können etwa

- die Zuweisung von Patienten durch das Krankenhaus ${ }^{16}$,

- das Letztentscheidungsrecht des Chefarztes $^{17}$,

14 LSG BW, NZS 2013, 501 (503); vgl. auch SG München, Urt. v. 10.3.2016, Az.: S 15R 1782/ 15.

15 LSG BW (4. Senat), MedR 2016, 222 (228).

16 SG Dortmund, das Krankenhaus 2006, 310 (311).

17 Vgl. LSG Nds-B, GesR 2016, 317 (319f.). 
- etwaige organisatorische Vorgaben durch den Krankenhausträger ${ }^{18}$,

- die Erbringung der ärztlichen Leistungen im Krankenhaus ${ }^{19}$,

- die Verpflichtung zur Zusammenarbeit mit dem Chefarzt der Abteilung sowie dem übrigen Personal ${ }^{20}$ oder

- die zeitlich begrenzte Nutzungsmöglichkeit der Ressourcen des Krankenhauses sein.

Unerheblich soll hingegen sein, dass der Arzt

- berechtigt gewesen ist, einzelne Aufträge abzulehnen ${ }^{21}$,

- keinen Einzelweisungen unterliegt und seine Arbeitszeit im Rahmen der betrieblichen Erfordernisse frei gestalten kann 22 oder

- hauptberuflich als niedergelassener Arzt selbstständig tätig ist und die Leistungserbringung je nach Bedarf an Wochenenden oder an Feier- und Brückentagen auf konsiliarärztlicher Basis erfolgt ${ }^{23}$.

Weiteres entscheidendes Kriterium ist nach der Rechtsprechung die Eingliederung des Arztes in den Krankenhausbetrieb. In diesem Kontext betonen die Gerichte bzw. stellen pauschal fest, dass der betreffende Arzt seine Leistungen stets in den Räumlichkeiten des Krankenhauses erbringt und auf die Zusammenarbeit mit dem angestellten Personal angewiesen ist, infolgedessen dieses Merkmal erfüllt sei ${ }^{24}$. Zum Teil wird darüber hinaus darauf abgestellt, dass

- der Arzt im OP-Plan/Dienstplan mitaufgenommen ist ${ }^{25}$,

- regelmäßig an bestimmten Tagen in der Woche tätig wird ${ }^{26}$,

- an ärztlichen Besprechungen und (gele-

18 Vgl. SG Darmstadt, Urt. v. 16.11.2015, Az.: S 8 KR 54/14.

19 SG Mannheim, Urt.v. 16.6.2011, Az.: S 15R 2545/09.

20 SG Dortmund, NZS 2015, 307 (308).

21 SG Darmstadt, Urt. v. 16.11.2015, Az.: S 8 KR $54 / 14$.

22 SG Dortmund, NZS 2015, 307 (309).

23 LSG BW, MedR 2016, 222.

24 Vgl. etwa SG Kassel, GesR 2013, 559 (564f.).

25 KH-Schiedsstelle Rheinland, Beschl. v. 12.1.2010, Verf.-Nr. 5/2010 S. 7.

26 Vgl. SG Münster, Urt.v. 18.12.2012, Az.: S 14R 180/12. gentlich) an Visiten teilnimmt ${ }^{27}$ oder

- zur Abstimmung mit anderen Ärzten verpflichtet ist ${ }^{28}$.

Als weitere Indizien für Annahme einer Stellung als Arbeitnehmer ziehen die Gerichte etwa

- die fehlende Verpflichtung zur Organisation einer Vertretung im Falle der eigenen Verhinderung ${ }^{29}$,

- die Zahlung eines erfolgsunabhängigen Stundenlohns ${ }^{30}$ oder

- die Verpflichtung zur persönlichen Leistungserbringung ${ }^{31}$ heran.

\section{Abweichende Beurteilung durch die arbeitsgerichtliche Rechtsprechung}

Demgegenüber fällt die arbeitsgerichtliche Rechtsprechung im Zusammenhang mit der Abgrenzung eines freien Dienstvertrages zu Arbeitsverträgen deutlich liberaler aus, die sich weitgehend an der Versorgungsrealität orientiert. Sie hebt hervor, dass die Einbindung in die Organisation der Klinik allein nicht ausschlaggebend sein könne, könne doch innerhalb der betrieblichen Abläufe die Leistung auf die eine oder die andere Weise erbracht werden, mithin abhängig oder selbständig. Damit gebe auch das Zeitregime keinen ausschlaggebenden Hinweis. ${ }^{32}$ Diese Auffassung teilt auch das LAG Hamm ${ }^{33}$ : „Die Tatsache, dass der Kläger seine Tätigkeit nur im Krankenhaus [...] verrichten konnte, spricht nicht zwingend für die Annahme eines Arbeitsverhältnisses, weil der Ort der Dienstleistung von der geschuldeten Tätigkeit abhängig ist und daher für die Rechtsnatur des Vertragsverhältnisses nicht entscheidend sein kann. Die Tatsache, dass der Kläger die ärztlichen Dienstleistungen nur [außerdem] nur zusammen mit den anderen Mit-

27 LSG NRW, Urt. v. 29.11.2006, Az.: L 11 (8) R $50 / 06$.

28 Vgl. LSG NRW, Urt. v. 30.9.2015, Az.: L 8 R $584 / 11$.

29 SG Darmstadt, Urt. v. 16.11.2015, Az.: S 8 KR 54/14; ebenso LAG RhPf, Beschl. v. 3.5.2010, Az.: 11 Ta 163/09.

30 LSG NRW, Urt. v. 30.9.2015, Az.: L 8 R 584/11.

31 SG Dortmund, NZS 2015, 307 (309).

32 Thür.LAG, Urt. v. 29.4.2010, Az.: 1 Ta 29/10.

33 LAG Hamm, Urt. v. 7.2.2011, Az.: 2 Ta 505/ 10; vgl. auch Hess.LAG, Urt. v. 14.1.2013, Az.: Sa $1213 / 12$. arbeitern [des Krankenhauses] sowie unter Verwendung der [dortigen] Einrichtung [...] erbringen sollte, lässt ebenfalls nicht zwingend auf das Vorliegen eines Arbeitsverhältnisses schließen. Denn auch sogenannte Beleghebammen und Belegärzte sind grundsätzlich keine Arbeitnehmer, obwohl auch sie die Einrichtungen eines Krankenhauses nutzen und mit anderen Mitarbeitern des Krankenhauses zusammen arbeiten (vgl. dazu BAG, Beschluss vom 21.02.2007 - 5 AZB 52/06, NZA 2007, 699 $[\ldots]) . “$

Ebenso sei es unbeachtlich, dass die Tätigkeit des Honorararztes zuvor durch einen angestellten Arzt ausgeübt wurde. Darüber hinaus weist das LAG Hessen ${ }^{34}$ darauf hin, dass ein vereinbartes Tageshonorar (im konkreten Fall EUR 1000,00) kein Direktionsrecht in zeitlicher Hinsicht indiziere. Dies gelte insbesondere auch dann nicht, wenn verbunden mit dem Tageshonorar ein Zeitfenster für die Tätigkeit, beispielsweise „max. 8:00 bis 16:00 Uhr, für Überstunden der durchschnittliche Stundensatz“, vereinbart wurde. Dadurch würde nicht die zeitliche Lage der Arbeitszeit verbindlich festgeschrieben, sondern vielmehr nur geregelt, für welche Tätigkeitsdauer das Tageshonorar anfallen solle. Selbst bei der Annahme einer Regelung der täglichen Dienstzeit, handele es sich dabei nicht um ein einseitiges Direktionsrecht des Arbeitnehmers, sondern um eine entsprechende einvernehmliche vertragliche Vereinbarung. Schließlich sieht das LAG Hessen ${ }^{35}$ zudem kein Argument für ein Arbeitsverhältnis darin, wenn die Vereinbarung zwischen Honorararzt und Krankenhaus unbefristet abgeschlossen wird. Durch eine unbefristete Gestaltung ergebe sich kein zwingender Hinweis für eine persönliche Abhängigkeit des Honorararztes.

\section{Kritische Würdigung}

Die abweichende Beurteilung durch die Arbeitsgerichte zeigt sehr deutlich die Schwächen der sozialgerichtlichen Rechtsprechung auf. Die Sozialgerichte verkennen vielfach, dass die Merkmale der Weisungsgebundenheit (insbesondere örtlichen und zeitlichen Weisungsgebundenheit) sowie

34 Hess.LAG, Urt. v. 14.1.2013, Az.: Sa 1213/12.

35 Hess.LAG, Urt. v. 14.1.2013, Az.: Sa 1213/12. 
der Eingliederung in die Betriebsorganisation bei einem Honorararzt nur bedingt geeignet sind, Rückschlüsse auf seinen Status zu erlauben. Die Nutzung der Räumlichkeiten und des (Hilfs-) Personals des jeweiligen Krankenhauses liegt in der Natur der Sache ${ }^{36}$ und ist geradezu typisch. Damit einhergehend und zur Gewährleistung einer optimalen Patientenbehandlung ist die Abstimmung mit den ärztlichen Kollegen und die Befolgung organisatorischer Vorgaben zwingende Voraussetzung. Es muss berücksichtigt werden, dass zeitliche und örtliche Absprachen im angemessenen Umfang notwendig sind, um einen reibungslosen organisatorischen Ablauf sicherzustellen - wie er im Übrigen auch in anderen selbstständigen Berufen zur Ausübung seiner Profession unabdingbar ist ${ }^{37}$.

Darüber hinaus begründet ein (fachliches) Weisungsrecht seitens des Krankenhauses gegenüber dem betreffenden Arzt sowie ein Letztentscheidungsrecht des Chefarztes nicht zwingend eine für das Arbeitsverhältnis typische Personalhoheit, da in der aufgabenorientierten Organisation des Krankenhauses die Schaffung einer betrieblichen Rangordnung mit einer abgestuften Aufgaben-, Kompetenz- und Verantwortungsstruktur einschließlich eines Kontroll-, Eingriffs- und Weisungsrechtes von oben nach unten ein unverzichtbares Erfordernis ist ${ }^{38}$. Mit einer Kontrolle der Qualität und Richtigkeit seiner Arbeit hat auch der freie Mitarbeiter zu rechnen. Eine arbeitnehmertypische Weisungsgebundenheit kann auch nicht daraus hergeleitet werden, dass der Arzt die zu behandelnden Patienten nicht frei wählen kann, denn die Zuweisung bestimmter Aufgaben im Rahmen einer nur abstrakt vereinbarten Tätigkeit ist auch gegenüber einem freien Mitarbeiter unerlässlich. Es handelt sich dabei um die notwendige Konkretisierung der von diesem zu erbringenden vertraglichen Leistung. Sie betrifft mithin nicht die „Ge-

36 Ebenso LAG RhPf, Beschl. v. 3.5.2010, Az.: 11 Ta 163/09; vgl. auch SG Berlin, Urt. v. 26.2.2014, Az.: S 208 KR 2118/12 („unabdingbar“); Korthus, das Krankenhaus 2012, 614 (617).

37 Vgl. auch SG Berlin, Urt. v. 10.2.2010, Az.: S 208 KR 102/09.

38 Genzel/Degener-Hencke, in: Laufs/Kern, HB d. Arztrechts, 4. Aufl. 2010, §84 Rn. 16. staltung“, sondern den Inhalt der geschuldeten Tätigkeit. ${ }^{39}$

Ebenso kann dem Kriterium des eigenen Unternehmerrisikos allenfalls eine untergeordnete Bedeutung beigemessen werden. Daher ist der Einsatz eigenen Kapitals bzw. eigener Betriebsmittel keine notwendige Voraussetzung für eine selbstständige Tätigkeit. Dies ist zum einen dem Umstand geschuldet, dass der Arzt im Krankhaus tätig wird und auf die Nutzung der dortigen Betriebsmittel angewiesen ist. Die Forderung nach einer finanziellen Gewinn- und Verlustbeteiligung ist daher abwegig ${ }^{40}$. Zudem ist zu bedenken, dass anderenfalls geistige oder andere betriebsmittelarme Tätigkeiten nie selbstständig ausgeübt werden könnten ${ }^{41}$.

Auf Grund ihrer überbordeten Anforderungen an die Freiberuflichkeit negiert die Rechtsprechung letztlich das Institut des Honorararztwesens, obgleich es sowohl vom Gesetzgeber in § 2 Abs. 1 S. 2 KHEntgG als auch vom $\mathrm{BGH}^{42}$ anerkannt wird. Sie legt vielfach derart strenge Maßstäbe an, dass der Status des (selbständigen) Honorararztes nur noch im Ausnahmefall bejaht werden kann. Ein derartiges Ergebnis läuft aber der höchstrichterlichen Rechtsprechung und dem Gesetz zuwider. Es erscheint daher vorzugswürdiger, im Rahmen der anzustellenden Gesamtabwägung andere Aspekte stärker in den Vordergrund zu rücken. In Betracht kommt insbesondere die Frage, in welchem Ausmaß der Arzt bei der Gestaltung seiner Dienste frei ist: Lage und Länge der Dienste, Möglichkeit der Ablehnung von Diensten, Zulässigkeit von Nebentätigkeiten/Bestehen einer Konkurrenzschutzklausel, zeitlicher Umfang der Tätigkeit sowie die Höhe der Gesamtvergütung. Weiterhin sollte stärker berücksichtigt werden, inwieweit der Arzt über das notwendige Maß, das für die Ausübung der Tätigkeit unerlässlich ist, hinaus in den Krankenhausbetrieb eingegliedert ist.

39 BAG, Urt. v. 5.7.2000, Az.: 5 AZR 888/98; vgl. auch Meier, MedR 2007, 709 (712).

40 So aber LSG Nds-B, GesR 2016, 317 (319).

41 Vgl. BSG, Urt. v. 30.10.2013, Az.: B 12 R 3 / $12 \mathrm{R}$.

42 BGH, MedR 2015, 120.

\section{Aktuelle Entscheidung des LSG Baden-Württemberg}

In einer aktuellen Entscheidung hatte das LSG Baden-Württemberg ${ }^{43}$ darüber zu befinden, ob die Tätigkeit einer Radiologin als Praxisvertreterin im Rahmen eines versicherungspflichtigen Beschäftigungsverhältnisses ausgeübt wurde. Dieses Urteil ist in zweifacher Hinsicht von besonderer Bedeutung. Zum einen stellt der erkennende Senat klarstellend fest, dass die Praxisvertretung gem. §31 Abs. 1 S. 2 Ärzte-ZV in rechtskonformer Weise grundsätzlich durch einen Honorararzt erfolgen kann. Jedoch begründet die Stellung als Praxisvertreterin nicht eo ipso das Vorliegen einer selbstständigen Tätigkeit. Sie stellt lediglich ein diesbezügliches Indiz dar.

Zum anderen sind der Entscheidung Wertungen zu entnehmen, die sich auf Honorarärzte im Krankenhaus gleichsam übertragen lassen. Im Gegensatz zu der eingangs dargestellten sozialgerichtlichen Judikatur legt das LSG Baden-Württemberg tendenziell liberalere Maßstäbe hinsichtlich der Statusbewertung an. Im konkreten Fall übernahm die Radiologin auf Grund mündlicher Vereinbarung Urlaubsvertretungen für die Praxisinhaber einer radiologischen Gemeinschaftspraxis, die sie frei bestimmen konnte. Inhalt ihrer Tätigkeit war die Befundung von radiologischen Untersuchungen (MRT, CT und Röntgen) in den Praxisräumen. Für ihre Tätigkeit stellte sie der Praxis Rechnungen nach einem vereinbarten Stundensatz von EUR 60,00. Ferner

- hatte sie für ihre Tätigkeit eine private Berufshaftpflichtversicherung abgeschlossen,

- haftete gegenüber Patienten für Behandlungsfehler,

- war in Dienstpläne und Urlaubsregelungen nicht eingebunden,

- konnte Aufträge ablehnen,

- durfte Dritte nicht beauftragen,

- hatte keinen einen Anspruch auf Entgeltfortzahlung oder Urlaubsvergütung,

- unterlag keiner Pflicht, an Dienstbesprechungen teilzunehmen,

- musste sich selbst um die Erfüllung der Fortbildungszeit kümmern, ohne hierfür Fortbildungszeit eingeräumt zu bekom-

43 LSG BW, Urt. v. 21.2.2017, Az.: L 11 2433/16. 
men und

- bekam keine Dienstkleidung gestellt.

Nicht zuletzt war die Radiologin für mehrere Praxen als Vertreterin tätig.

Andererseits

- bestand ein Weisungsrecht gegenüber nichtärztlichem Personal,

- erfolgte die Befundung in einer festen Reihenfolge,

- wurden die Befunde von einem Praxisinhaber gegengelesen,

- war sie mit denselben Aufgaben betraut, die üblicherweise von angestellten Ärzten wahrgenommen wurden, und

- war an den Betriebskosten nicht beteiligt.

In Übereinstimmung mit der Vorinstanz bejahte das LSG Baden-Württemberg eine selbstständige Tätigkeit. Nach Ansicht des Gerichts besitzt in diesem Kontext weder die Weisungsfreiheit in fachlicher Hinsicht noch eine gewisse Eingliederung des betreffenden Arztes Aussagekraft in die eine oder die andere Richtung: Qua Gesetz unterliegt der Arzt weitestgehend keiner fachlichen Weisungsgebundenheit, die nur bei der Eingliederung in Hierarchien durchbrochen wird. Die eigenverantwortliche Befundung durch die Radiologin entspricht daher dem Wesen der ärztlichen Tätigkeit und kann als solche nicht zur Beurteilung beitragen. Aus der Natur der Sache besteht außerdem eine gewisse Eingebundenheit, da die Tätigkeit in den Praxisräumen in Abstimmung sowie in Zusammenarbeit mit dem (nicht-) ärztlichen Personal ausgeübt werden muss. Diese äußere Eingliederung in die Organisationsstruktur der Praxis liegt allerdings in der Natur der Sache der Tätigkeit und ist daher unvermeidlich. Demzufolge kann sie ebenfalls kein valides Abgrenzungskriterium darstellen.

Stattdessen stellt der erkennende Senat maßgeblich darauf ab, ob und inwieweit die Radiologin über das zwingend notwendige Maße hinaus in die Betriebsorganisation der Gemeinschaftspraxis eingegliedert war und einer nicht-fachlichen Weisungsgebundenheit unterlag. Im vorliegenden Fall verneinte er im Rahmen einer Gesamtabwägung die Unselbstständigkeit ihrer Tätigkeit, da sie nicht in vergleichbarem
Maße wie die angestellten Ärzte eingegliedert war. So

- mussten die angestellten Ärzte ein elektronisches Zeiterfassungssystem nutzen,

- ihnen wurden für die Befundung bestimmte Protokolle verbindlich vorgegeben,

- sie mussten an Teambesprechungen teilnehmen,

- ihnen wurde Kleidung mit dem eingestickten Namen und dem Logo der Praxis gestellt und

- sie nahmen an sozialen betrieblichen Veranstaltungen teil, zu denen die Radiologin als Praxisvertreterin nicht eingeladen wurde.

Schließlich überwachten und steuerten die Praxisinhaber die zur Einhaltung des Facharztstandards vorgeschriebenen ärztlichen Fortbildungspflichten ausschließlich bei den angestellten Ärzten.

Ein wichtiges Indiz für das Vorliegen einer selbständigen Tätigkeit war außerdem, dass der Radiologin keine festen Arbeitszeiten oder Schichten ohne vorherige Absprache und gegen ihren Willen zugewiesen werden konnten. Vielmehr stand es ihr frei, an welchen Tagen sie eine Urlaubsvertretung übernehmen wollte. Ohne nähere Begründung nahmen die Richter - wenn auch nur in geringem Maße - ein für Selbständigkeit sprechendes Unternehmerrisiko an. Maßgebendes Kriterium für ein solches Risiko ist, ob eigenes Kapital oder die eigene Arbeitskraft auch mit der Gefahr des Verlustes eingesetzt wird, der Erfolg des Einsatzes der sächlichen oder persönlichen Mittel also ungewiss ist. Zudem erscheint es nach ihrer Ansicht nicht sachgerecht, allein aufgrund des vereinbarten pauschalen Stundenhonorars von EUR 60,00 ein unternehmerisches Risiko auszuschließen, da sich eine stundenweise Abrechnung viel eher anbietet als eine aufwendig zu berechnende Vergütung nach einzelnen ärztlichen Diensten entsprechend der Gebührenordnung für Ärzte oder nach dem Einheitlichen Bewertungsmaßstab für ärztliche Leistungen unter Abzug von Praxiskosten. Die Vergütung ist daher als neutrales Kriterium zu sehen.

Auf die Frage, ob die Radiologin in die Hierarchie der Gemeinschaftspraxis eingeglie- dert war, ging das Gericht in seinen Urteilsgründen zwar nicht mehr ein. Jedoch lässt sich allein aus dem Umstand, dass die Radiologin die von ihr erstellten Befunde mit einem der Praxisinhaber abstimmen musste, keine unselbstständige Tätigkeit herleiten. Es handelt sich insoweit um eine generelle Qualitätssicherungsmaßnahme, die keine Aussage zu einem Über-/Unterordnungsverhältnis enthält.

Abschließend weist das LSG Baden-Württemberg unter Berufung auf das BSG ${ }^{44}$ daraufhin, dass in Zweifelsfällen der tatsächliche Wille der Vertragsparteien maßgeblich ist, sofern dieser Wille den festgestellten sonstigen tatsächlichen Verhältnissen nicht offensichtlich widerspricht und er durch weitere Aspekte gestützt wird bzw. die übrigen Umstände gleichermaßen für Selbstständigkeit wie für eine Beschäftigung sprechen.

\section{Fazit}

Trotz der gewichtigen Argumente gegen die eingangs dargestellte sozialgerichtliche Judikatur verbleiben erhebliche Rechtsunsicherheiten in Bezug auf die Beurteilung und Bejahung der Selbstständigkeit von Honorarärzten, denn eine höchstrichterliche Entscheidung, die konkrete Maßstäbe zu deren Statusbewertung aufstellt, steht bislang noch aus. Vielmehr ist zum einen die Tendenz zu beobachten, dass die Klageverfahren, die in der ersten Instanz mit der Bestätigung der Freiberuflichkeit enden, in der zweiten Instanz aufgehoben werden ${ }^{45}$. Zum anderen ist zu konstatieren, dass die (sozial-) gerichtliche Rechtsprechung infolge einer pauschalen Bejahung des Beschäftigungsbegriffs mehrheitlich zu der Annahme neigt, der Honorararzt werde nicht selbständig tätig ${ }^{46}$. Exemplarisch lässt sich dies an einem Urteil des LSG Niedersachsen-Bremen ${ }^{47}$ aus der jüngeren Vergangenheit belegen.

Auf Grund der anzustellenden Gesamtabwägung lassen sich ferner klare Rechtspre-
44 Schnapp, NZS 2014, 41 (42). 
chungslinien kaum erkennen und rechtssicher aufstellen. Stattdessen ist die Beurteilung durch eine Vielzahl von Einzelkriterien und Indizien geprägt. Merkmale für ein abhängiges Beschäftigungsverhältnis sind etwa: zeitliche und organisatorische Vorgaben sowie diesbezügliche Einbindung; Vergütung nach festem Stundensatz; Weisungsgebundenheit gegenüber den Praxisgesellschaftern; Supervision durch Praxisgesellschafter; Einbindung in die Hierarchie; Auftreten als Mitarbeiter der Praxis gegenüber den Patienten; Zusammenarbeit mit Mitarbeitern der Praxis; kein unternehmerisches Risiko oder Chance; kein Kapitaleinsatz; keine Beteiligung an Betriebskosten; Ausübung der Tätigkeit in fremder Arbeitsorganisation; Abrechnungen mit Patienten über das Krankenhaus bzw. die Praxis. Für eine selbstständige Tätigkeit spricht hingegen: Möglichkeit zur Ablehnung einzelner Aufträge; keine Verpflichtung zur Teilnahme an Dienstbespre- chungen; deutlich über dem Angestelltengehalt liegendes Honorar; keine Stellung von Arbeitskleidung und keine Verpflichtung zum Tragen einheitlicher Kleidung.

Generell gilt, dass das Risiko der Verneinung einer selbständigen Tätigkeit steigt, je mehr die Stellung des Honorararztes der eines angestellten Arztes gleicht. Daher ist anzuraten, den Arzt nur insoweit in den Krankenhausbetrieb bzw. die Praxis einzugliedern, wie es für die Ausübung der ärztlichen Tätigkeit unerlässlich ist. Diese Sichtweise teilt - im Einklang mit der herrschenden arbeitsgerichtlichen Rechtsprechung - nunmehr auch das LSG BadenWürttemberg, welches sich verstärkt mit der Versorgungsrealität auseinandergesetzt hat. Es bleibt daher zu hoffen, dass sich seine Rechtsauffassung zukünftig durchsetzen wird. Angesichts der grundlegend differierenden Rechtsprechungslinien sei abschließend daraufhin gewiesen, dass die Sozialgerichte bei der Prüfung sozialrechtlicher Fragestellungen nicht an die arbeitsrechtliche Beurteilung der Arbeitnehmereigenschaft gebunden sind, sodass voneinander abweichende Beurteilungen der beiden Gerichtsbarkeiten im Hinblick auf die Statusfrage von Honorarärzten denkbar sind.

Prof. Dr. Peter Wigge,

Rechtsanwalt

Fachanwalt für Medizinrecht

Dipl. jur. Jan Harald Schütz, LL.M., Wiss. Mitarbeiter

Rechtsanwälte Wigge

Scharnhorststraße 40

48151 Münster

$++49 / 251 / 535950$

++49/251/5359599

kanzlei@ra-wigge.de

www.ra-wigge.de 